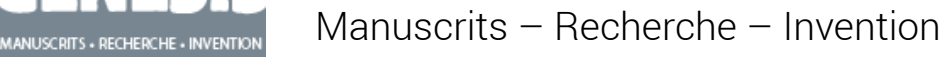

$48 \mid 2019$

Écritures Jeunesse

\title{
Christian Voltz, Heu-reux
}

\section{Karine Meshoub-Manière}

\section{(2) OpenEdition}

Journals

Édition électronique

URL : http://journals.openedition.org/genesis/4164

DOI : 10.4000/genesis.4164

ISSN : 2268-1590

\section{Éditeur :}

Presses universitaires de Paris Sorbonne (PUPS), Société internationale de génétique artistique littéraire et scientifique (SIGALES)

\section{Édition imprimée}

Date de publication : 1 juin 2019

Pagination : 135-144

ISSN : 1167-5101

\section{Référence électronique}

Karine Meshoub-Manière, «Christian Voltz, Heu-reux », Genesis [En ligne], 48 | 2019, mis en ligne le 01 juin 2020, consulté le 25 janvier 2021. URL : http://journals.openedition.org/genesis/4164 ; DOI : https://doi.org/10.4000/genesis.4164 


\title{
Christian Voltz, Heu-reux
}

\author{
Karine Meshoub-Manière
}

$\mathrm{C}$ hristian Voltz, auteur et illustrateur d'une quarantaine d'albums pour la jeunesse, a su imposer son univers aux milieux spécialisés et au grand public. En effet, il réalise ses illustrations en volume à partir de montage et d'assemblage de matériaux de récupération : vis, écrous, fils de fer, laine, bois, tissus, cuir, objets divers et variés, boulons, etc. sortis de son atelier qui ressemble davantage à un garage, à un atelier de réparateur ou encore d'électricien (fig. 1) qu'à un bureau d'écrivain.

Soucieux de partager son univers, singulier et animé par une forme d'engagement politique (celui de la récupération, de la lutte contre le gaspillage, de la décroissance), il n'hésite pas à aller à la rencontre de son public et à partager à travers plusieurs expositions son processus de création :

- «De Bric et d'Broc» (2012), exposition réalisée par L'Imagier Vagabond autour de six albums de l'auteur (cinq pour lesquels il est auteur et illustrateur et un pour lequel il est illustrateur) ${ }^{1}$, comprenant 23 originaux et des panneaux techniques présentant tout le processus de travail préparatoire de l'auteur-illustrateur;

- «Les petites personnes de Christian Voltz», exposition réalisée par L'Imagier Vagabond avec atelier de création graphique autour de son album Dans l'atelier de Christian Voltz (Éditions du Rouergue, 2011).

- «Christian Voltz, poésie bidouillée», au Colysée de Lambersart de décembre 2016 à février 20172, rassemblant deux expositions : la première, «la Fabric», un «joyeux bazar» de pièces disparates ressemblant à des vieux jouets ramenés à la vie par un savant fou, et la seconde, «les trésors minuscules », illustrations, gravures, sculptures, céramiques, créée en 2006 à l'initiative

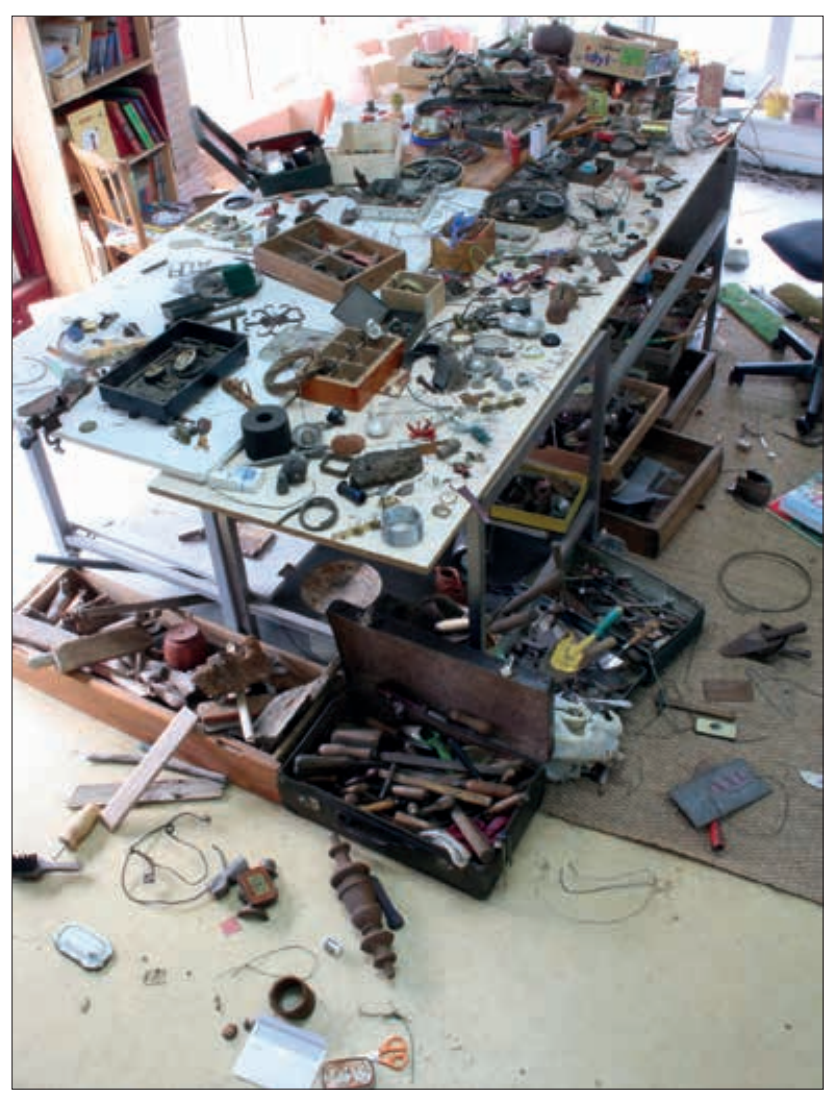

Fig. 1 : «Bazar table»*.

de l'équipe de la médiathèque Émile Aillaud de Bobigny. Plusieurs panneaux présentaient le travail de l'artiste du brouillon à la réalisation de l'album avec un certain nombre d'avant-textes reproduits.

(*) La dénomination des figures est celle que Christian Voltz a choisie pour identifier chacun de ses documents.

1. C'est pas ma faute, texte et illustrations de Christian Voltz, Éditions du Rouergue, 2002. — La caresse du papillon, texte et illustrations de Christian Voltz, Éditions du Rouergue, 2005. — Le livre le plus génial que j'ai jamais lu, texte et illustrations de Christian Voltz, Pastel, 2008. — Sacré sandwich, texte et illustrations de Christian Voltz, Pastel, 2006. — Un aigle dans le dos, texte et illustrations de Christian Voltz, Éditions du Rouergue, 2003. - Drôle de nuit, texte d'Ernst Jandl, illustrations de Christian Voltz, Rue du monde, 2008.

2. Dossier de presse de l'exposition : http://www.eulalie.fr/IMG/pdf/dossier_de_presse_christian_voltz.pdf, consulté le 18 décembre 2017. 
Le dossier génétique que nous allons présenter comprend dix documents autour de l'album Heu-reux ${ }^{3}$ (Éditions du Rouergue, 2016) dont les reproductions nous ont été aimablement fournies par l'auteur :

- un document autour du travail sur le personnage (correspondant à la page 3 de la version éditée 4 de l'album) (fig. 5 infra);

- deux extraits consécutifs d'un même état du chemin de fer réalisé sur un cahier format A4 à lignes anglaises ${ }^{5}$ documents que nous présenterons plus en détail (fig. 6 et 7 infra);

- deux photographies de l'atelier au moment de la construction des personnages en volume, avec matériaux de recyclage, ici celui de Grobull et du prince (fig. 8 et 9 infra);

- deux photographies rendant compte de la construction de l'œuvre dans son ensemble à partir de la maquette provisoire et de sa transformation en volume, avant que chaque double page ne soit prise en photo par Jean-Louis Hess (fig. 10 et 11 infra);

- une photographie du photographe en action (fig. 12 infra). Il réalise, à ce moment-là, les ektachromes, clichés qui seront ensuite scannés pour qu'ils puissent être retouchés au besoin (au niveau de la lumière surtout) et sur lesquels sera inséré le texte avec choix de la typographie (selon le plan de la maquette).

Cette construction de l'illustration en volume est tout à fait singulière, c'est elle qui fait la signature de l'auteur et permet au lecteur de reconnaître ses œuvres au premier coup d'œil.

\section{Présentation de l'œuvre Heu-reux}

Publié aux Éditions du Rouergue en 2016, qui revendiquent un certain engagement en faveur de la créativité, dans les sujets abordés et dans les formes proposées, Heu-reux, album de quarante pages au format $23 \mathrm{~cm} \times$ $21 \mathrm{~cm}$, aborde avec humour et légèreté la question de l'homosexualité. Le roi Grobull, «taureau-tyran craint et respecté», décide de marier son fils unique, le prince Jean-Georges. Et comme «l'épouse n'a pas encore été choisie», le roi lance un appel à toutes les vaches du royaume afin que son fils puisse choisir sa bien-aimée librement. Le défilé commence, mais aucune des prétendantes ne trouve grâce aux yeux du prince, qui confie son désarroi à ses acolytes, Hubert le Bélier et Ginette la «belle poulette ». Ces amis le poussent à dire la vérité à son père, mais le prince n'y parvient pas. Restant sur son idée de lui trouver chaussure à son pied, le roi fait alors organiser un nouveau défilé, de truies, cette fois. Même schéma : aucune ne convient au prince. Puis défilé de juments, brebis et chèvres. Même schéma. Le roi s'énerve et accuse le prince de n'avoir d'yeux que pour sa comparse la poule, puis, même si l'union lui paraît étrange, il consent à laisser son fils choisir son élue, la chose importante pour lui étant qu'il soit «heu-reux». Le fils se saisit de la proposition et demande à son père s'il peut vraiment choisir qui il veut. Le roi acquiesce et Jean-Georges lui annonce vouloir épouser... Hubert le bélier. «Et donc, ils se marièrent, vécurent heureux et... et voilà !»

\section{Le chemin de fer de Heu-reux}

Le chemin de fer permet à l'auteur, dans son processus de création, «d'avoir une vision d'ensemble du livre et de voir si le déroulement de l'histoire est bien fluide ${ }^{6}$ ». Il le conçoit comme «les pages du livre, dessinées les unes après les autres, en petit ${ }^{7} »$. Cependant, quand on observe ce qu'il nomme «chemin de fer», l'on ne peut qu'être surpris de voir que ce dernier ne représente pas véritablement la maquette

3. Christian Voltz, Heu-reux, Arles, Éditions du Rouergue, 2016.

4. Nous appelons «version éditée» l'état du texte et de l'image retenu pour l'édition.

5. Nous avons interrogé l'auteur, par échange de courriers électroniques, sur sa pratique du cahier : «K.M.-M. : Le chemin de fer de Heu-reux est écrit sur un cahier. Est-ce un cahier dédié à la même œuvre? Si oui, y a-t-il des pages dédiées à l'élaboration de l'histoire (notes, recherches, idées en vrac, etc.) ou bien faites-vous ce travail préparatoire ailleurs (dans votre tête, dans des [petits] carnets, sur des feuilles volantes, etc.) ? - C.V. : C'est un cahier qui n'est pas dédié à une seule œuvre. J'y note mes idées, dessine d'après nature, fais des chemins de fer... à la suite. Mais je travaille aussi sur des feuilles volantes et je ne garde pas grand-chose de ces travaux préparatoires » (propos recueillis le 5 septembre 2018).

6. Propos de l'auteur recueillis sur les panneaux explicatifs de son processus créatif pour l'exposition «Christian Voltz, poésie bidouillée», Lambersart, de décembre 2016 à février 2017.

7. Ibid. 


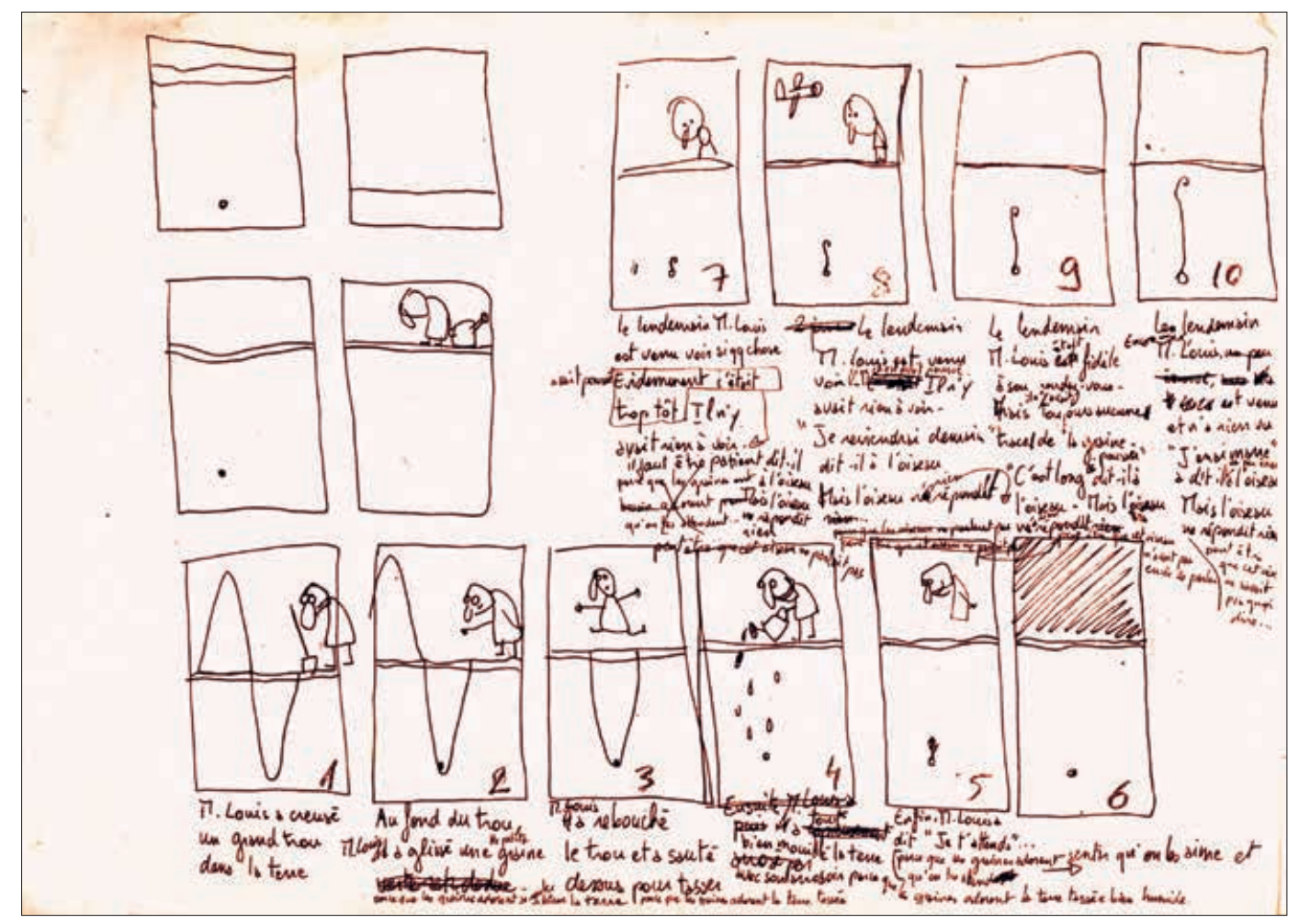

Fig. 2 : Chemin de fer de Toujours rien.

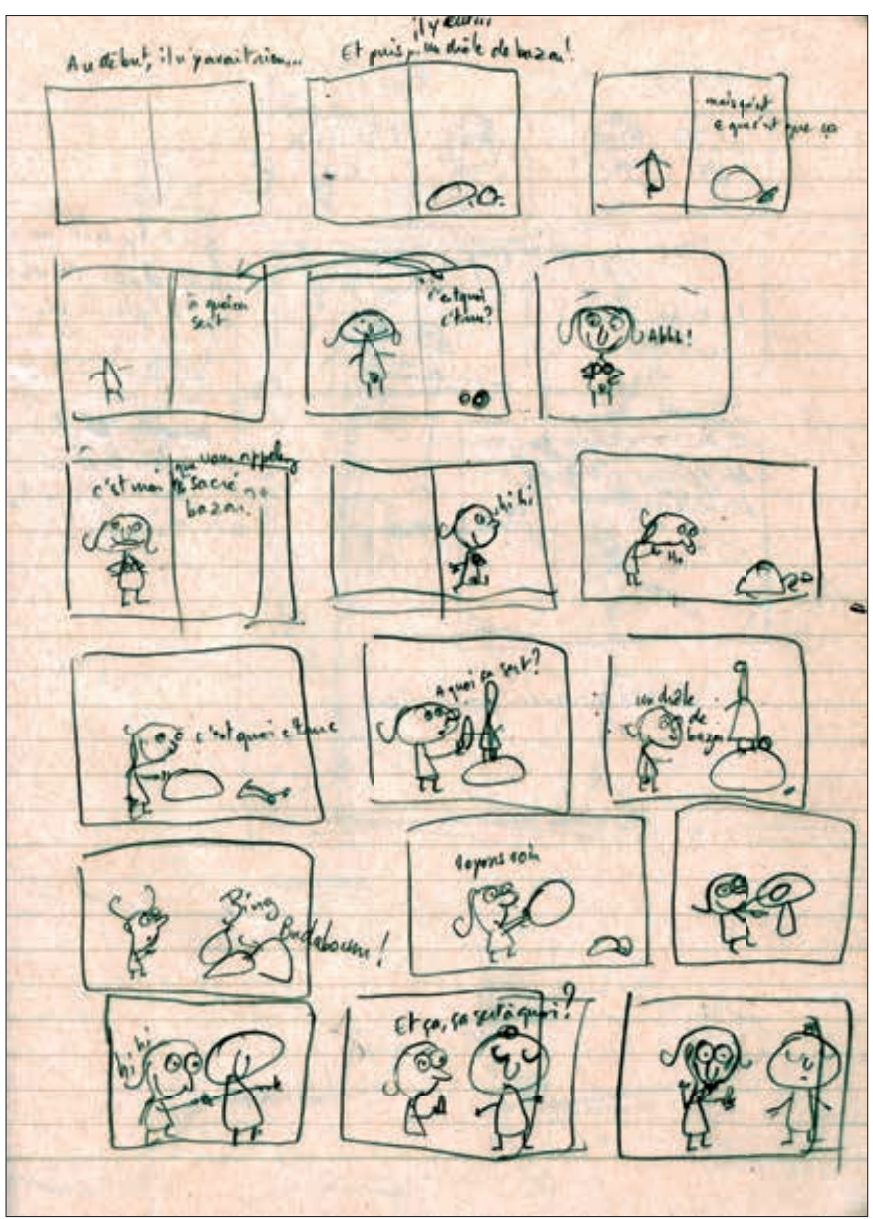

Fig. 3 : Chemin de fer de Il est où. 


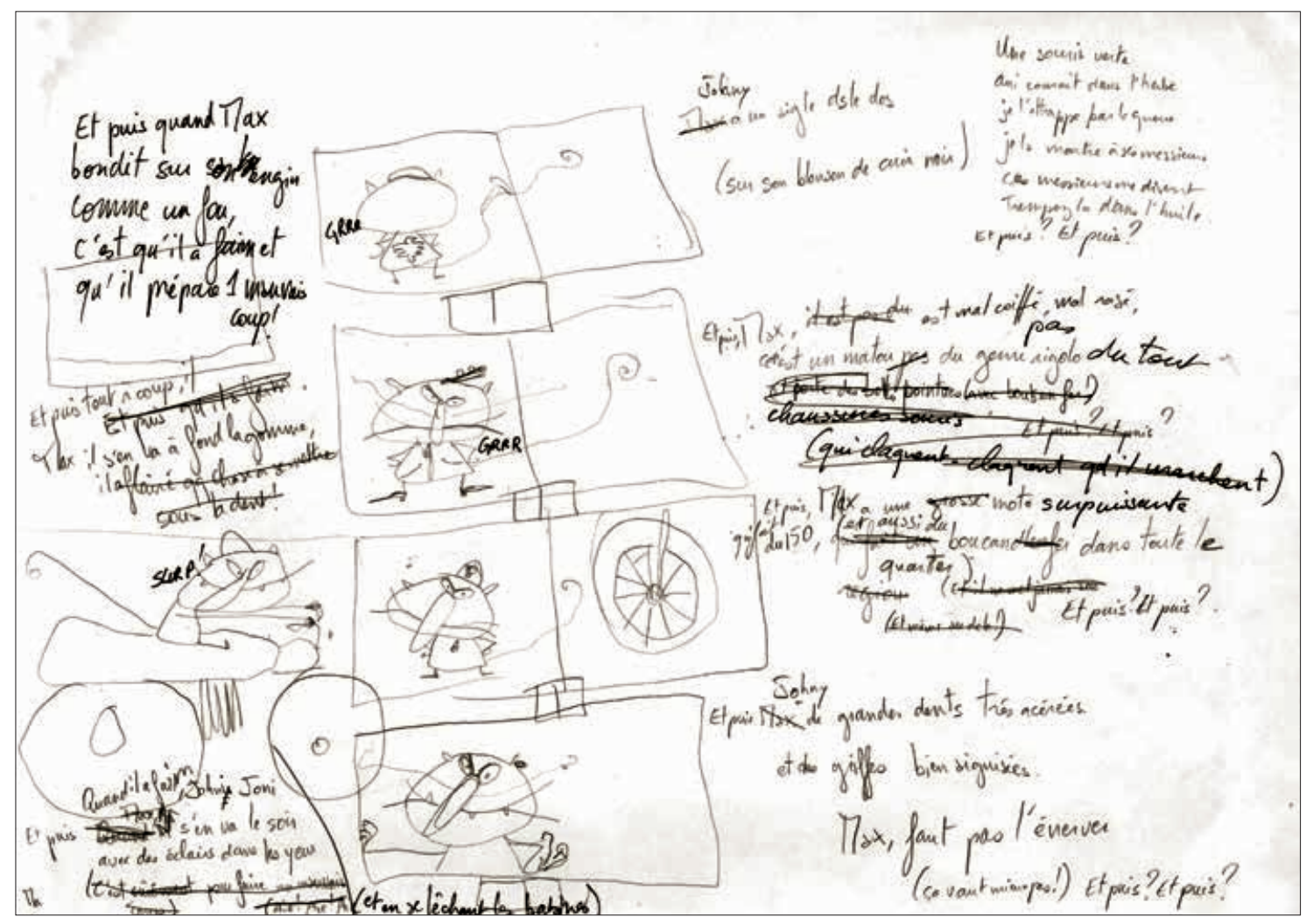

Figure 4 : Chemin de fer de Un aigle dans le dos («Max»).

de l'œuvre à venir. En effet, l'illustration et le texte ne sont pas réunis sur le même espace de la double page tel qu'il figurera dans la version éditée.

Parmi les reproductions des différents chemins de fer que l'auteur nous a confiées ${ }^{8}$, texte et illustrations sont séparés :

- dans le chemin de fer de Heu-reux qui nous intéresse ici (cf. fig. 6 et 7 infra), l'illustration, réalisée dans un cadre, est située à gauche de la page du cahier, le texte est à droite ou en dessous de l'illustration;

- dans le chemin de fer de Toujours rien (fig. 2), réalisé sur une page $\mathrm{A} 4$ au format paysage, on observe en bas de page une première série d'illustrations, côte à côte, numérotées de 1 à 6 avec le texte manuscrit en dessous, puis au-dessus de cette première série sur la droite de la page, une deuxième série d'illustrations numérotées de 7 à 10, elles aussi avec le texte manuscrit figurant en dessous;

- dans le chemin de fer de Il est où (fig. 3), réalisé sur la page $\mathrm{A} 4 \mathrm{~d}$ 'un cahier à lignes anglaises au format portrait, figurent dix-huit cases matérialisant les doubles pages de l'album (la pliure n'étant pas systématiquement marquée). Pour les deux premières doubles pages, le texte est écrit au-dessus d'elles, en dehors donc de l'espace du livre; pour d'autres, le texte se trouve à l'intérieur de l'espace de la double page; certaines sont sans texte;
- dans le chemin de fer intitulé par l'auteur «Max» (fig. 4), sur une page A4 au format paysage, sont matérialisées quatre doubles pages de l'album contenant l'illustration. Elles occupent le deuxième tiers de la feuille avec, de part et d'autre, différents états du texte (repérables grâce aux changements d'encre).

Ces variations dans la construction et la conception du chemin de fer laissent entrevoir que rien n'est encore achevé et que la succession des illustrations peut avoir une influence sur le texte et entraîner des modifications, comme le confirme l'auteur :

Là encore (i.e. au moment de la réalisation du chemin de fer), je supprime du texte et j'essaie de ne garder que l'essentiel pour arriver à un juste équilibre textes/images 9 .

La première double page du chemin de fer de Heu-reux (fig. 6 infra) contient quatre étapes du récit :

- un premier face à face entre le père (Grobull) et le fils (page 11) après le défilé de toutes les vaches du royaume;

8. Nous avons en notre possession une reproduction du chemin de fer de Toujours rien, Il est où, Un aigle dans le dos («Max») en plus de celle de Heu-reux que nous présentons ici.

9. «Christian Voltz, poésie bidouillée», ibid. 
- la convocation du Premier Conseiller par le roi pour qu'il lance l'invitation à toutes les truies du royaume, à la manière d'un héraut ;

- le défilé des nouvelles prétendantes devant le prince qui les congédie toutes;

- un nouveau face-à-face entre le père et le fils à la fin de ce second défilé.

La seconde double page du chemin de fer (fig. 7 infra) contient la suite du récit en cinq étapes :

- le roi ordonne à son conseiller de lancer un appel à toutes les «brebis, juments, ânesses et chèvres » du royaume;

- le défilé des candidates, sans succès aucun auprès du prince;

- l'embarras du prince qui ne peut se résoudre à choisir une candidate qu'il ne peut aimer;

- la réaction emportée du roi qui pense avoir compris pour qui bat le cœur de son fils, sa comparse la poule, avant qu'il ne concède à son fils de choisir qui il veut pourvu qu'il soit heureux;

- la surprise et la joie du prince devant la proposition de son père.

La fin de l'histoire telle qu'on peut la lire dans la version éditée était-elle présente dans cet état du texte? Les documents à notre disposition ne nous permettent pas de le savoir. Il en va de même pour le début présent dans la version éditée (l'annonce au royaume du mariage et le premier défilé des vaches).

\section{L'articulation texte/image}

Christian Voltz ne cesse d'affirmer la prééminence de l'histoire sur l'illustration, à chaque fois qu'il accepte de présenter son mode de création :

Tout commence par l'histoire. L'histoire, c'est le cœur du livre. Sans histoire, pas de personnage, pas de début, ni de fin. Une histoire peu intéressante donnera toujours un livre inintéressant, même si les illustrations sont magnifiques! C'est pourquoi, je passe beaucoup de temps à écrire, barrer, écrire gommer, écrire, m'énerver, et réécrire encore mon histoire... jusqu'à ce que j'estime qu'elle puisse être racontée. D'ailleurs, la plupart de mes histoires resteront à l'état de brouillon, au fond d'un tiroir... 10
Même s'il est toujours délicat et dangereux pour le généticien de se fier aux déclarations d'un auteur sur son processus de création, nous tentons ici l'hypothèse d'une chronologie fondée sur les propos de Christian Voltz où le chemin de fer serait l'étape qui a suivi l'écriture de l'histoire. Le texte n'en est pas pour autant figé, comme en témoignent les nombreuses modifications (biffures, ratures, déplacement, ajouts) sur lesquelles nous reviendrons.

La configuration de la page nous donne à penser que, dans cette phase de travail, l'auteur commence par l'illustration, le texte venant trouver sa place à droite de l'illustration ou en dessous. Les corrections empiètent, à plusieurs reprises, sur le cadre de l'illustration.

L'illustration, réalisée à grands traits avec le même stylo que l'écriture du texte, occupe tout l'espace de la page matérialisé par un rectangle au trait noir fait à main levée : il semblerait que l'organisation du texte sur la double page de l'album vienne dans un second temps, une fois le texte et le contenu de l'illustration arrêtés, contrairement à Agnès Rosenstiehl (cf. étude supra) qui construit ses chemins de fer sur le modèle de la maquette de l'œuvre à venir (seize cases correspondant aux trente-deux pages de l'album édité) avec une répartition texte/image posée dès le début.

Quelques éléments remarquables permettent d'interroger l'articulation du texte et de l'image dans la réalisation du chemin de fer de l'œuvre : la permanence des personnages (dans leur caractérisation et leur désignation); les changements d'énonciation qui privilégient l'adresse à l'autre ; les modifications du (et dans le) texte entraînées par la deuxième double page (chemin de fer 1 ).

10. Propos diffusés sur les panneaux de l'exposition «Christian Voltz, poésie bidouillée », Lambersart de décembre 2016 à février 2017. Ou encore ici, au cours d'un entretien réalisé par Sylvie Neeman, pour la revue Parole en 2011, lorsqu'elle l'interroge sur la naissance de ses livres : «S.N. : Comment naît un livre ? Est-ce tel nez (telle brosse...) qui va évoquer tel trait de caractère chez un personnage et en fonction de ce trait de caractère vous allez imaginer une histoire ? Quelle est l'impulsion ? C.V. : Non, non, le point de départ, c'est toujours l'histoire, l'histoire est toujours première. D'abord, je l'écris, ensuite je fais des dessins, c'est là que je cherche les caractères, les expressions, le physique des personnages, qui est très important. Ensuite seulement je prends mon fil de fer, mes objets, et c'est alors la vraie recherche du ou des personnages, la concrétisation. Et cette étape-là peut être longue !», dans Sylvie Neeman, «Christian Voltz, l'artisan de l'éphémère durable», revue Parole, $\mathrm{n}^{\circ} 3$, 2011 (Institut suisse Jeunesse et Médias). 
Sur l'ensemble du chemin de fer (fig. 6 et 7 infra), il n'y a pas encore d'unité dans le dessin des personnages : Grobull a tantôt des taches noires, tantôt un pelage uni ; tantôt un anneau dans les naseaux, tantôt non. Il est cependant marqué et identifiable par la forme de ses cornes, plus épaisses et plus grandes que celles du prince, par exemple, mais aussi par son regard menaçant, exprimant la colère, l'exaspération (quand il s'adresse à son fils) ou la domination (quand il s'adresse à son conseiller). Dans les essais que fait l'auteur pour trouver l'image finale de son personnage, l'on retrouve l'anneau nasal et le regard menaçant de Grobull (fig. 5 infra).

Même absence d'unité pour le prince, tantôt au pelage tacheté, tantôt uni. Son regard exprime la timidité devant l'autorité forte de son père et la gêne mêlée de honte quand ses refus suscitent l'emportement du père. L'identité visuelle des personnages secondaires est clairement posée : l'âne pour le conseiller, le bélier et la poule pour les deux acolytes du prince.

Le flottement sur les noms est intéressant. Tout d'abord, il y a une rature sur le nom du roi : «Grosbull» devient «Grobull », un «b » venant remplacer en surépaisseur «sb». Le jeu de mots contenu dans le nom du personnage (où «bull» en anglais signifie «mâle») n'a pas besoin du renfort de l'orthographe pour fonctionner, l'image acoustique du nom propre suffit à le faire advenir. Le prince s'appelle dans cet état du texte «Jean René» au lieu de «Jean-Georges» dans la version éditée. L'intention du double prénom marquant de façon stéréotypée la noble lignée du héros est présente dès le début. Dans la tradition onomastique, Georges incarne la figure de la timidité, de la réserve là où René serait la figure du tyran ou du séducteur. Autre hypothèse : étymologiquement, «Georges» signifie «le laboureur», ce qui sied à un bovidé par métonymie : il porte le nom de l'une de ses fonctions dans la ferme, assurer le labour en traînant la charrue. Enfin, les acolytes du prince se nomment Hector pour le bélier (au lieu d'Hubert) et Violette pour la poule (au lieu de Ginette).

Dans le texte, le langage du prince est modifié dès la première illustration : «désolé, père », réponse à l'interrogation de Grobull sur son rejet de toutes les vaches du royaume, est remplacé par « «Je crains bien que non, mon père», répond doucement le fiston» qui fait écho à la réponse du fils quand la scène se répète après le défilé des truies, trois doubles pages plus loin : ««Il semble bien que non», répond timidement le fiston »; l'écho se retrouve dans la réaction du père également.

Pour la deuxième illustration (Grobull sommant son conseiller de lancer un second appel) et le texte qui l'accompagne, une flèche vient indiquer leur déplacement après un autre épisode, qui, lui n'a pas d'illustration : il s'agit des propos de Grobull qui justifient le nouvel ordre qu'il va donner à son conseiller (convoquer les truies du royaume pour satisfaire au bonheur de son fils, quoi que cela lui en coûte, ici le non-respect de la race et de la lignée). Comment interpréter ce déplacement? Peut-être comme un élément venant étayer l'antériorité du texte sur l'illustration, la séquence illustrée correspondant à une partie infime du texte et demandant à être complétée en amont par une autre illustration qui viendrait l'annoncer.

Le texte, qui est alors sans illustration, subit des modifications dans la forme du discours du roi : quand il énonce la déception que représente le choix de son fils de rejeter toutes les vaches du royaume, il s'adresse à son conseiller et parle de son fils à la troisième personne, dans une forme d'aparté, lui refusant ainsi le statut de destinataire direct de son propos ( $\ll \mathrm{J}$ 'aurais préféré que mon fils se marie avec une représentante de sa race, mais l'important est que»); dans la seconde version, l'auteur change de système énonciatif et réécrit au style direct le propos du roi, avec interpellation du fils : «Fils, j'aurais préféré que tu te maries avec une représentante de ta notre race, mais l'important est que tu sois heureux, mon fils!». Notons que cette adresse directe au fils se retrouve après le troisième défilé, quand le roi s'emporte en imaginant que «(son fils) n'(a) d'yeux que pour cette vulgaire poulette! » auprès de laquelle il a tenté de chercher du réconfort (dans la double page précédente).

Quand le roi somme à nouveau son conseiller d'aller chercher une nouvelle vague de prétendantes (la troisième, première double page du chemin de fer 2 qui se situe, dans la chronologie générale, trois doubles pages plus loin que le premier épisode), ce dernier n'est pas nommé dans un premier temps, mais identifiable dans le sujet de l'injonction avec l'emploi du pronom indéfini «on» («Qu'on cherche donc les brebis, les juments et les ânesses et les chèvres, hurle le roi»). L'auteur ajoute après coup «au 1 er conseiller» après «hurla le roi», et il ajoute également avant le texte qui accompagne la double page suivante («On fit donc 
chercher les plus belles femelles des races brebis, juments, anesse (sic) et chèvres pour les présenter au prince. ») : «Bien parlé, sir, dit le 1er conseiller (quel fayot celui-là !)» et modifie la suite ainsi :

\section{fait venir \\ On fit done chercher \\ et il \\ les plus belles...}

L'illustration, quant à elle, ne correspond pas à l'adresse du roi à son conseiller comme c'était le cas dans la deuxième double page du chemin de fer 1, mais à la série des prétendantes - brebis, juments, ânesses et chèvres - où seules apparaissent clairement jument et ânesse, une troisième tête est esquissée, probablement la chèvre, mais rien n'est sûr.
L'analyse de ces deux chemins de fer nous a permis de mettre au jour la façon dont s'articulent, dans le processus de création de Christian Voltz, le texte et l'image et plus particulièrement l'effet que la présence de l'illustration peut avoir sur le texte. Si l'auteur est convaincu que «ce qui est dit par l'image n'a plus besoin de l'être par les mots ${ }^{11}$ » et qu'il peut à ce titre supprimer du texte, la lecture de ses documents de genèse nous invite à observer l'inverse : l'illustration peut entraîner l'auteur à ajouter du texte, à donner de l'épaisseur à l'ensemble en créant des échos dans le texte, portés et permis par l'illustration.

11. Propos recueillis par Élodie Soulié, dans son article «Des héros de plumes et de boulons », pour le journal Le Parisien du 26 novembre 2007.

\section{Le dossier génétique de Heu-reux - illustrations}

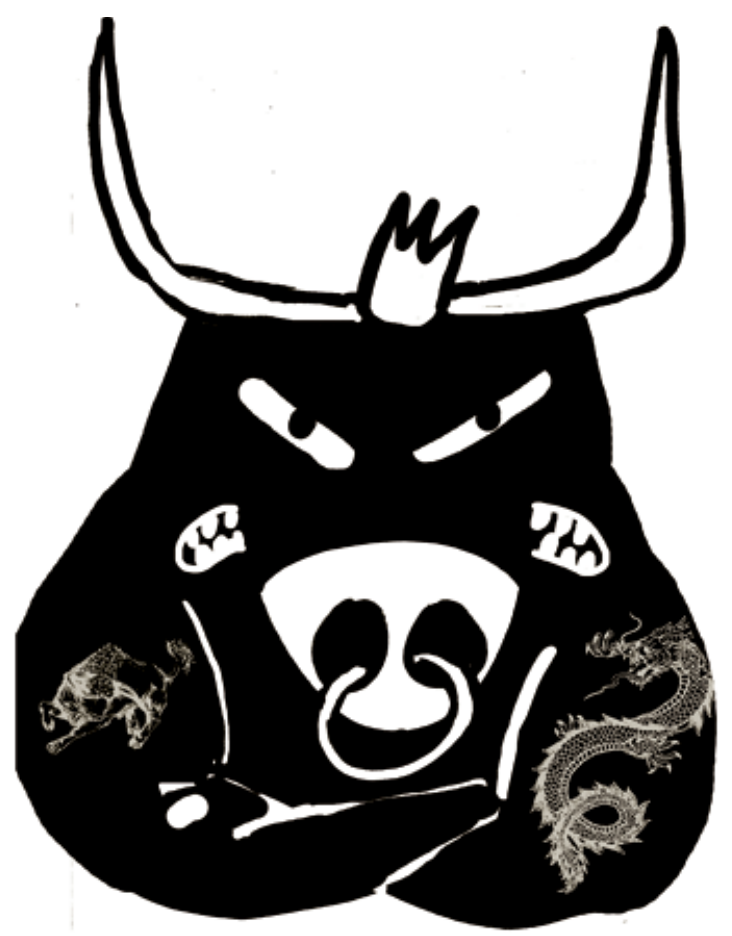

Fig. 5 : Travail sur personnage «Grobull». 
Fig. 6 : Chemin de fer 1 , format $21 \mathrm{~cm} \times 29,7 \mathrm{~cm}$.

Fig. 7 : Chemin de fer 2 , format $21 \mathrm{~cm} \times 29,7 \mathrm{~cm}$
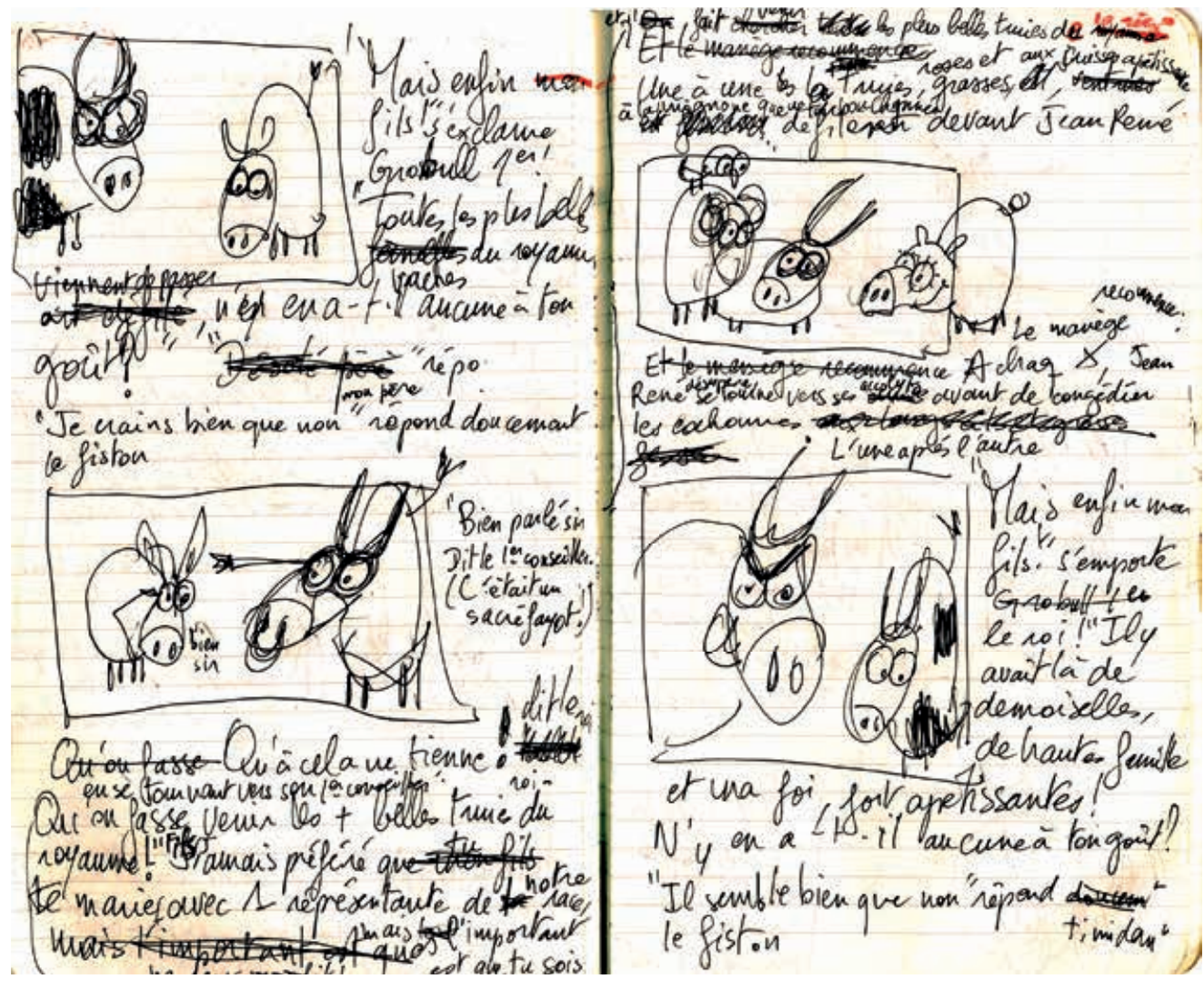
les cahomis i L'uneapes l'autre (1) Maid enfinman et una foi for a pretssantes! $N_{y}^{\prime}$ en a L. it au cureà tongoif? "Il semblle bien que non "repend le fiston timidan"

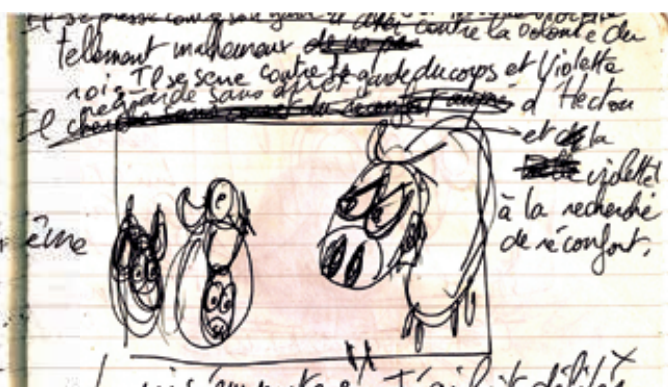

we roi s'emporte: I'ai air defiles

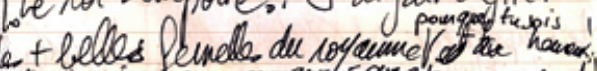

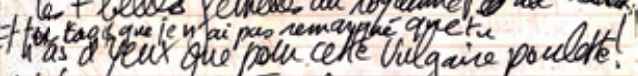
Etbien soift. Il hucle a present:

"Je le plas inipoctant st que te sois hemoux. IJ Jet'or do une déthe henenx!" Chasis qui ta venx ef quion en finisse?

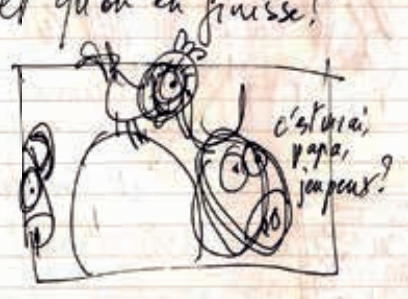




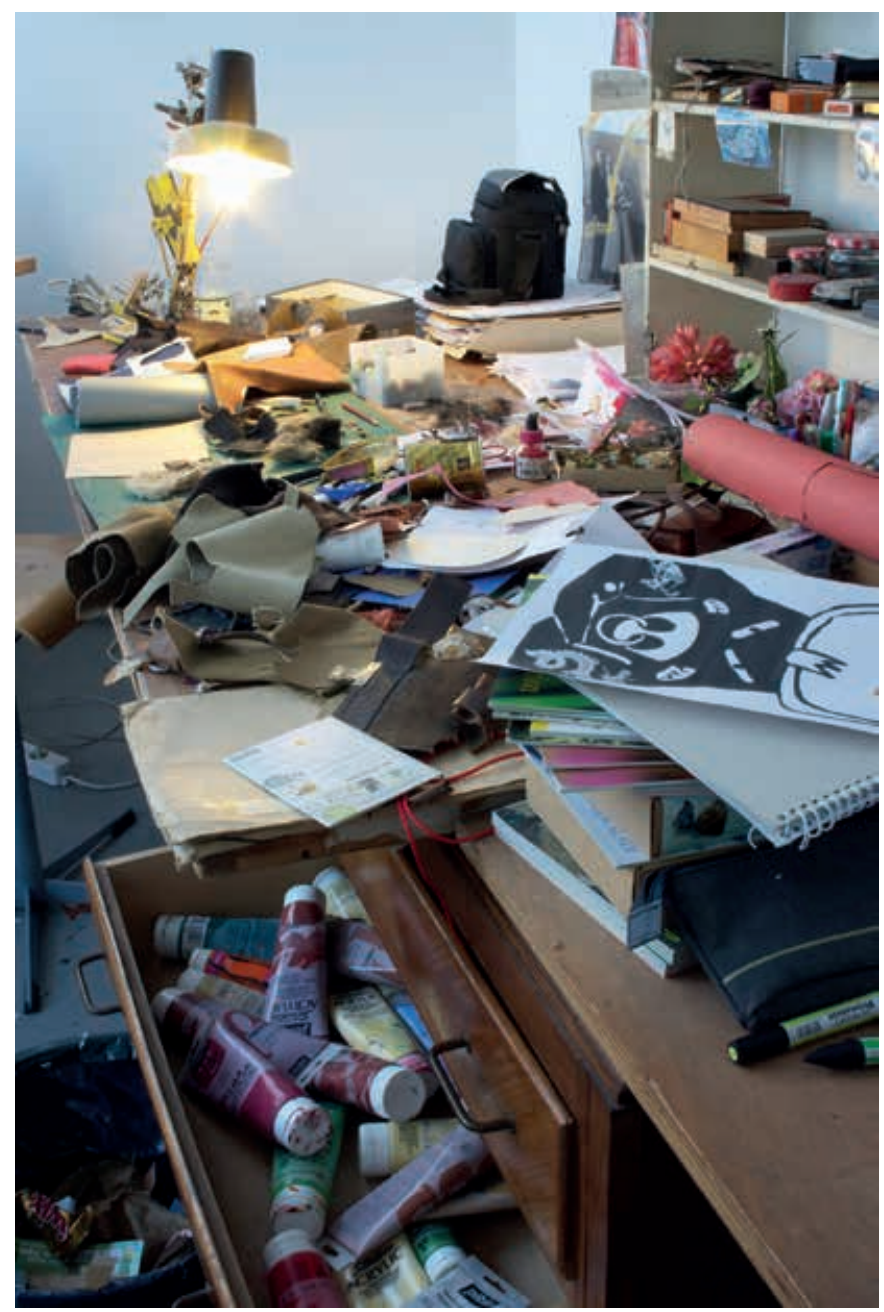

Fig. 8 : Construction personnage «Grobull».

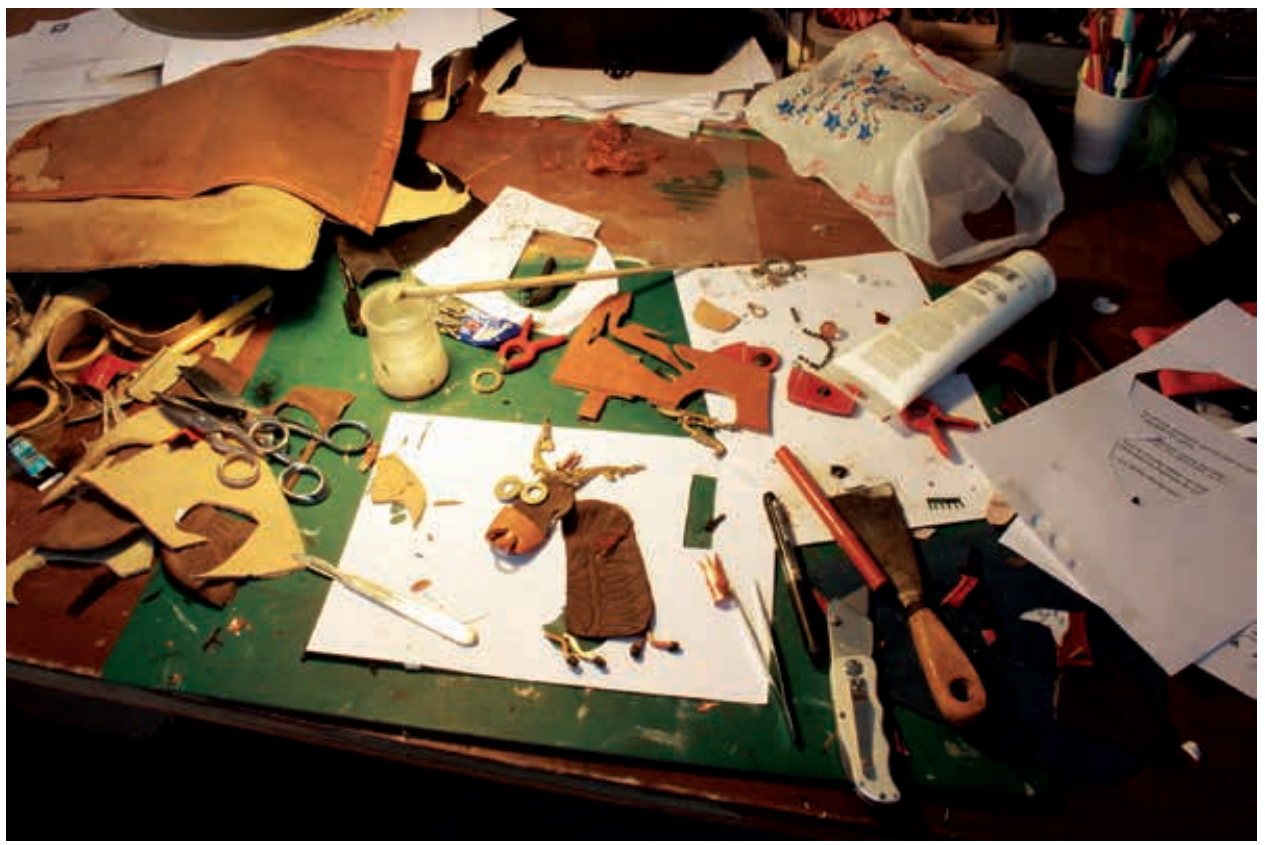

Fig. 9 : Construction personnage «Prince». 


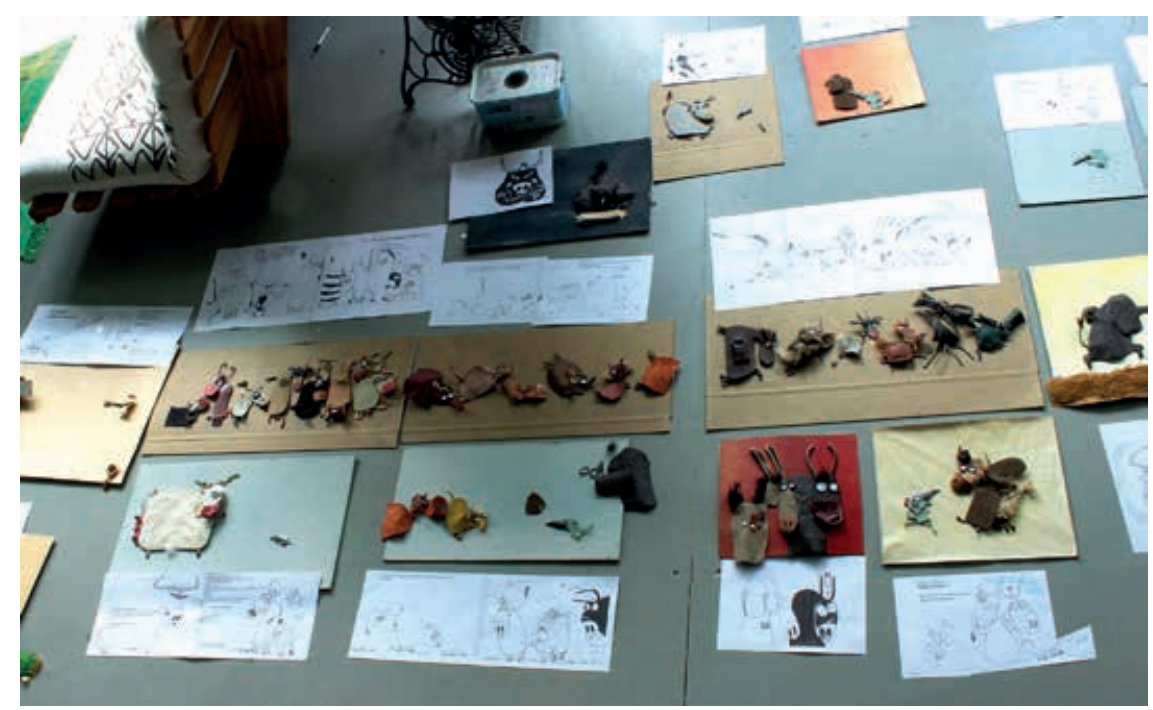

Fig. 10 : Toutes les pages sont prêtes.
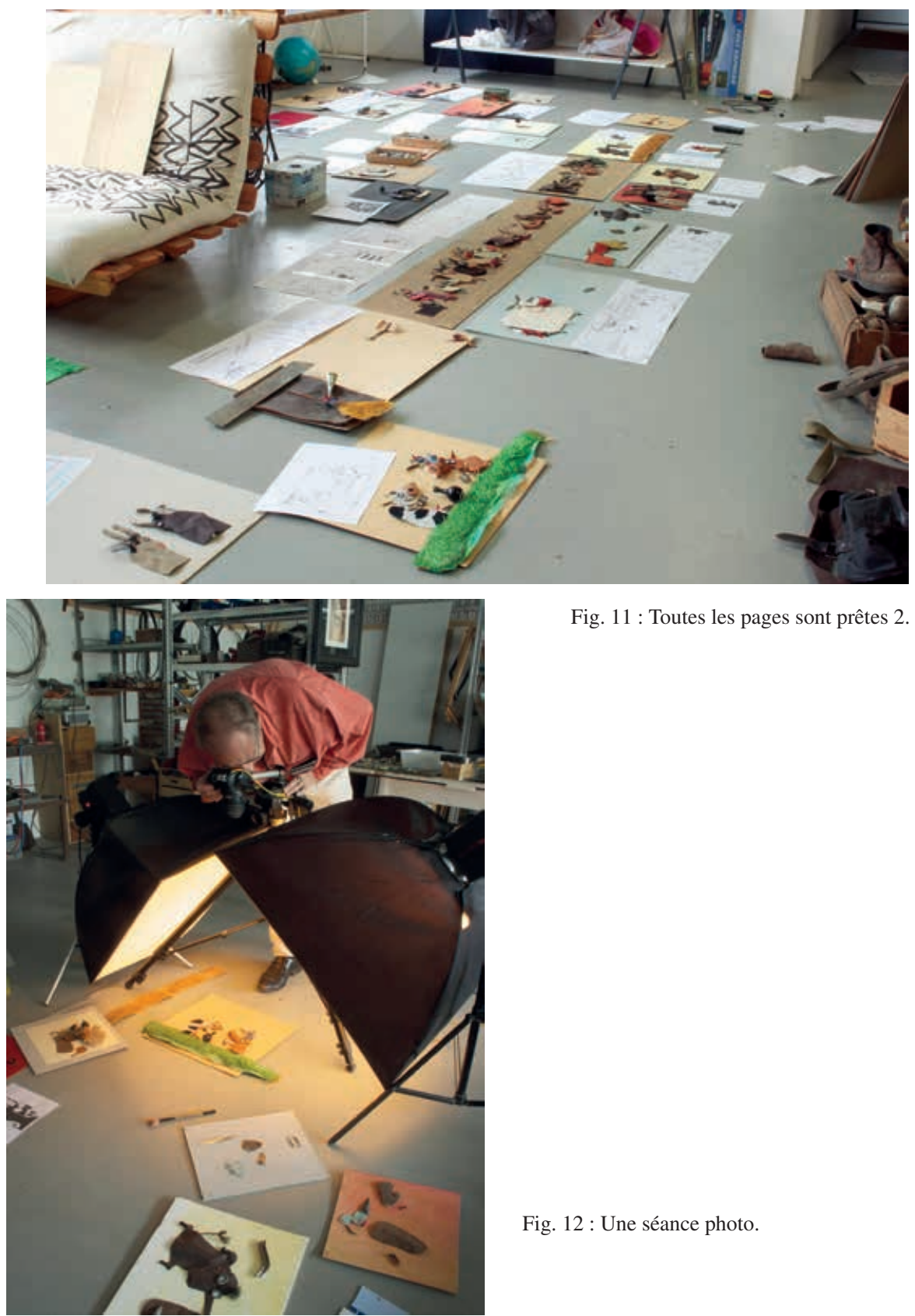

Fig. 11 : Toutes les pages sont prêtes 2 .

Fig. 12 : Une séance photo. 